of the cell cycle studied by the method of classical TEM in the cells of the meristematic zone of the wheat root. The transformations of peripheral chromosomal material (PCM) in telophase, nucleolonema of nucleoli in interphase and prophase, chromatin transformation (condensation and decondensation) under conditions of the geomagnetic field, weakened magnetic field and static magnetic field at low positive temperatures and salinity were investigated and analyzed. Using an analysis of the dynamics of structural modifications of proliferating nuclei, there was a disturbance in the formation of the chromonema and condensed chromatin in the middle interphase, a change in the structural organization of the nucleolus during the transition from late interphase to prophase, as well as a change in the structure of PCM, pre-nucleolus and chromatin in telophase. A scheme has been proposed for the formation of a specific nucleolonemal and chromonemal structure and its influence on the structural organization of chromosomes in anaphase and telophase. The nature of this structure identified earlier in normal and stressful conditions in rye and barley $[1,2]$ and wheat in the acidic and alkaline reaction of the environment and cold $[3,4]$ is discussed. The studied effects associated with the transformation of the structural organization of the nuclei caused a change in the expression level of the genes of some DNA methyltransferases, probably related to the epigenetic regulation of the genome. The proposed mechanisms of action of low positive temperatures, salinization, magnetic field on the nuclear compartment and its domains, regulation of chromatin decondensation, export of pro-ribosomal particles under stress, and the fine structure of the nucleolonema are discussed.
This work was supported by the Russian Science Foundation (grant 18-016-00150) and by the state assignment AAAA-A18-118051890089-0.

Referenses: 1.Baranova EN, Baranova GB, Kharchenko PN (2011) Effect of weak magnetic field and low positive temperature on chromatin and nucleolus ultrastructure of rye and barley. Russ Agric Sci 37: 453-461. 2. Wang J, Zhang F (2015) Nucleolus disassembly and distribution of segregated nucleolar material in prophase of root-tip meristematic cells in Triticum aestivum L. Arch Biol Sci 67: 405-410. 3. Avetisova LV, Kadykov VA (1985) Ultrastructure of Wheat Apical Meristem Cells at Low Positive Temperatures, 1: Nuclear Structure. Tsitologiya, 27: 28-32. 4. Baranova EN, Gulevich AA (2009) Structural organization of nuclei and nucleoli of wheat shoot and root meristem during germination under alkaline $\mathrm{pH}$ conditions. Russ Agri Sci 35: 11-14.

doi: http://dx.doi.org/10.7124/bc.0009D7

\section{B-5. The Perichromatin Region: a crossroad of events}

\author{
M. Biggiogera, S. Siciliani, L. Zannino \\ Dipartimento di Biologia e Biotecnologie "L. Spall- \\ anzani", University of Pavia, Italy \\ marcobig@unipv.it
}

The Perichromatin Region (PR) is the 200-nmthick rim surrounding the condensed chromatin areas in the interphase cell nucleus. First described by Bernhard (1969), PR has been studied for years and several fundamental processes have been shown to take place there: DNA replication, repair, transcription, co-transcriptional splicing. Recently we have pointed out that possibly other mechanisms occur in that region (Masiello et al. 2018). We have studied the PR in several cell and tissue models, both in physiological conditions and after drug treatment. We can confirm that not only methylation on cytosine in DNA and RNA 
occurs there (Masiello and Biggiogera, 2017) but also demethylation of $5 \mathrm{mC}$ leading to $5 \mathrm{hmC}$ formation. We have detected by EM immunocytochemistry the presence of DNMT3 as well as TET enzymes within this area. Our data strengthens the hypothesis that PR represents one of the most active nuclear areas, where the active focus is mobile. Since only particular conditions allow its study, it will be challenging to work on $\mathrm{PR}$ in vivo via super resolution.

References: Bernhard W (1969) A new staining procedure for electron microscopical cytology. J Ultrastruct Res 27(3):250-265 Masiello I, Biggiogera M (2017) Ultrastructural localization of 5-methylcytosine on DNA and RNA. Cell Mol Life Sci 74:3057-3064 Masiello I, Siciliani S, Biggiogera MHistochem Cell Biol (2018) 150:227-233

doi: http://dx.doi.org/10.7124/bc.000A06

\section{B-6. Testing the biological significance of the nuclear localization of actin}

Péter Borkúti ${ }^{1}$, Izabella Bajusz ${ }^{1}$, Csaba Bajusz $^{1}$, Ildikó Kristó ${ }^{1}$, Zoltán Kovács ${ }^{1}$, Péter Vilmos ${ }^{1}$

\footnotetext{
${ }^{1}$ Biological Research Centre of the Hungarian Academy of Sciences, Szeged, Hungary borkutip@brc.hu
}

In recent years it has become clear that the main cytoskeletal component of eukaryotic cells, Actin is present also in the nucleus. However, there was no attempt so far to investigate the biological significance of this localization therefore, we don't know today how essential is nuclear Actin for the organism. The aim of our work is to understand the importance of nuclear Actin in Drosophila melanogaster. Methods: Mutagenesis via RS-elements, molecular cloning, transgenic animal generation, fly genetics, immunohistochemistry. Results: Out of the six Actin coding genes of Drosophila melanogaster, two (Act5C and Act42A) encode ubiquitously expressed, essential proteins which are also present in the nucleus. We chose Act5C in our experiments because its lack is lethal, and the gene has a simple structure, small size and well characterized regulation. To investigate the biological significance of Actin's nuclear function at the organism level, we created a two-component genetic system of Act5C. One element of this system is an Act5C null-mutant line which lacks the whole protein coding region, and the other element is represented by animals carrying various $\mathrm{Act} 5 \mathrm{C}$ transgenes. The transgenes express different forms of the Act5C protein including one which is tagged with a Nuclear Export Signal (NES). The NES tag dramatically decreases the amount of nuclear Act5C, without damaging the protein's cytoplasmic functions. Our subsequent experiments revealed that the NES-Act5C transgene is able to rescue the lethal phenotype of the nullmutants suggesting that the nuclear localization of Actin is not essential. However, the efficiency of the rescue was apparently lower than in the control experiments. Conclusion: Based on this finding and other results, we hypothesize that Actin has essential role in the nucleus but its nuclear functions are most likely redundantly secured. The ways through which the nuclear functions of Actin are assured are under investigation and will be discussed. 